\title{
Investigation of Toxin Gene Diversity, Molecular Epidemiology, and Antimicrobial Resistance of Clostridium difficile Isolated from 12 Hospitals in South Korea
}

Heejung Kim, M.D. ${ }^{1}$, Seok Hoon Jeong, M.D. ${ }^{1}$, Kyoung Ho Roh, M.D. ${ }^{2}$, Seong Geun Hong, M.D. ${ }^{3}$, Jong Wan Kim, M.D. ${ }^{4}$, Myung-Geun Shin, M.D. ${ }^{5}$, Mi-Na Kim, M.D. ${ }^{6}$, Hee Bong Shin, M.D. ${ }^{7}$, Young Uh, M.D. ${ }^{8}$, Hyukmin Lee, M.D. ${ }^{9}$, Kyungwon Lee, M.D. ${ }^{1}$

Department of Laboratory Medicine and Research Institute of Bacterial Resistance', Yonsei University College of Medicine, Seoul; Department of Laboratory Medicine ${ }^{2}$, Korea University College of Medicine, Seoul; Department of Laboratory Medicine ${ }^{3}$, CHA University, Seongnam; Department of Laboratory Medicine ${ }^{4}$, Dankook University Hospital, Cheonan; Department of Laboratory Medicine ${ }^{5}$, Chonnam National University Medical School, Gwangju; Department of Laboratory Medicine ${ }^{6}$, University of Ulsan College of Medicine and Asan Medical Center, Seoul; Department of Laboratory Medicine”, Soonchunhyang University College of Medicine, Bucheon; Department of Laboratory Medicine $^{8}$, Yonsei University Wonju College of Medicine, Wonju; Department of Laboratory Medicine ${ }^{9}$, Kwandong University College of Medicine, Goyang, Korea

Background: Clostridium difficile is a major cause of antibiotic-associated diarrhea. The objective of this study was to characterize clinical isolates of $C$. difficile obtained from various regions in Korea with regard to their toxin status, molecular type, and antimicrobial susceptibility.

Methods: We analyzed a total of 408 C. difficile isolates obtained between 2006 and 2008 from 408 patients with diarrhea in 12 South Korean teaching hospitals. C. difficile toxin genes tcdA, tcdB, $c d t A$, and $c d t B$ were detected by PCR. Molecular genotyping was performed by PCR ribotyping. Antimicrobial susceptibilities of the $120 \mathrm{C}$. difficile isolates were assessed by agar dilution methods.

Results: Among 337 toxigenic isolates, 105 were toxin A-negative and toxin B-positive $\left(A^{-} B^{+}\right)$and 29 were binary toxin-producing strains. PCR ribotyping showed 50 different ribotype patterns. The 5 most frequently occurring ribotypes comprised $62.0 \%$ of all identified ribotypes. No isolate was susceptible to cefoxitin, and all except 1 were susceptible to piperacillin and piperacillin-tazobactam. The resistance rates of isolates to imipenem, cefotetan, moxifloxacin, ampicillin, and clindamycin were $25 \%, 34 \%, 42 \%, 51 \%$, and $60 \%$, respectively. The isolates showed no resistance to metronidazole or vancomycin.

Conclusions: This is the first nationwide study on the toxin status, including PCR ribotyping and antimicrobial resistance, of $C$. difficile isolates in Korea. The prevalence of $A^{-} B^{+}$strains was $25.7 \%$, much higher than that reported from other countries. Binary toxin-producing strains accounted for $7.1 \%$ of all strains, which was not rare in Korea. The most prevalent ribotype was ribotype 017 , and all $\mathrm{AB}^{+}$strains showed this pattern. We did not isolate strains with decreased susceptibility to metronidazole or vancomycin. (Korean J Lab Med 2010;30:491-7)

Key Words : Clostridium difficile, toxin A, toxin B, Ribotyping, Drug Resistance, Epidemiology

Received: June 17, 2010

Revision received: August 9, 2010

Accepted: August 12, 2010

Corresponding author: Kyungwon Lee, M.D.

Department of Laboratory Medicine, Research Institute of Bacterial Resistance, Yonsei University College of Medicine, 134 Sinchon-dong, Seodaemun-gu, Seoul 120-752, Korea Tel : +82-2-2228-2446, Fax : +82-2-313-0908 E-mail : leekcp@yuhs.ac

*This work was supported by the Korea Research Foundation Grant funded by the Korean Government (MOEHRD, Basic Research Promotion Fund) (KRF-2007-313-E00440).

ISSN 1598-6535 (C) The Korean Society for Laboratory Medicine

\section{INTRODUCTION}

Clostridium difficile is a major cause of nosocomial antibiotic-associated diarrhea [1]. Toxins A (enterotoxin; TcdA) and B (cytotoxin; TcdB) are well-known primary virulence factors of $C$. difficile [2]. These toxins are encoded by 2 separate genes, tcd $A$ and $t c d B$, which are located 
in the pathogenicity locus of the chromosome called PaLoc $[3,4]$.

Toxigenic isolates of $C$. difficile usually produce both toxins $\mathrm{A}$ and $\mathrm{B}$. Toxin $\mathrm{A}$-negative and toxin B-positive $\left(\mathrm{A}^{-} \mathrm{B}^{+}\right)$strains of $C$. difficile were first described in the early 1990s $[5,6] . \mathrm{A}^{-} \mathrm{B}^{+}$strains fail to produce detectable amounts of toxin $\mathrm{A}$ due to a deletion in the repeating sequence of the tcdA gene. However, $\mathrm{A}^{-} \mathrm{B}^{+}$strains have been associated with clinical conditions ranging from asymptomatic carriage to fatal pseudomembranous colitis. Alfa et al. [7] reported convincing evidence that indicates that these strains have been responsible for outbreaks in hospitals.

Some isolates of $C$. difficile produce an additional binary toxin (actin-specific ADP-ribosyltransferase toxin, CDT), whose role in $C$. difficile-associated disease (CDAD) is unclear [8]. The 2 genes cdtA and cdtB encode the enzymatic (CDTa) and binding (CDTb) components of the binary toxin. These genes are located on the CDT locus of the chromosome but are not part of the PaLoc [8, 9]. The prevalence of $\mathrm{A}^{-} \mathrm{B}^{+}$, and binary toxin-producing $C$. difficile strains varies geographically [10].

Recently, outbreaks of CDAD due to an emerging strain of $C$. difficile (PCR ribotype 027) associated with high morbidity and mortality have been reported in Canada, the United States, and Europe [11]. This strain produces a binary toxin and has deletions in $t c d C$, a putative negative regulator for toxins A and B [11, 12]. The epidemic strain is resistant to gatifloxacin and moxifloxacin, and increasing use of fluoroquinolone has been considered a risk factor in these outbreaks [11]. The most commonly used drugs for the treatment of CDAD are metronidazole (MTZ) and vancomycin (VAN). C. difficile is considered to be susceptible to both agents, and therefore, the in vitro activity of these agents against $C$. difficile isolates is rarely performed in most centers. However, a few reports have been published regarding elevated minimum inhibitory concentrations (MIC) of MTZ and VAN against $C$. difficile [13]. Moreover, increased resistance to antimicrobial agents has played a role in their selection in hospital environments [14].

The objective of this study was to characterize clinical isolates of $C$. difficile associated with diarrhea throughout South Korea with regard to their toxin status, molecular typing, and antimicrobial susceptibility.

\section{MATERALS AND METHODS}

\section{Bacterial strains}

We obtained and analyzed 408 unduplicated isolates of C. difficile recovered between 2006 and 2008 from 408 patients with diarrhea in 12 tertiary teaching hospitals in 7 regions of Korea. We received $C$. difficile isolates or frozen stool samples from all 12 hospitals. Stool samples were cultured anerobically on $C$. difficile selective agar (CDSA, Becton Dickinson and Company, Sparks, MD, USA) for $48 \mathrm{hr}$ at $37^{\circ} \mathrm{C}$. Species identification was performed on the basis of typical morphology on agar plates as well as characteristic odor and ATB 32A system results (BioMerieux SA, Marcy I'Etoile, France). The reference strains VPI 10463, 3608/03, SE844, 48489, 1470, and UK078 were supplied by Dr. Maja Rupnik, Michel Delmee, and Thomas V. Riley.

\section{Toxin analysis by PCR}

C. difficile toxin genes were detected by PCR as described previously $[15,16]$. The primer pairs used were NK9-NK11 for the repetitive domain of tcdA, NK104NK105 for tcdB, cdtA pos-cdtA rev for cdtA, and cdtB pos-cdtB rev for $c d t B$.

\section{PCR ribotyping}

PCR ribotyping was performed as previously described with the primers $5^{\prime}$-CTGGGGTGAAGTCGTAACAAGG$3^{\prime}$ (position 1445 to 1466 of the 16S rRNA gene) and 5' GCGCCCTTTGTAGCTTGACC-3' (position 20 to 1 of the 23S rRNA gene) [17]. Comparison of the PCR ribotyping patterns was performed visually. Ribotype patterns that differed by at least 1 band were assigned to different types. Ribotype groups were designated by upper- and 
lower-case letters combined with a number.

\section{4. $t c d C$ sequencing}

The tcdC gene was PCR-amplified with the primers PaL15 and PaL16 on the ribotype 027 strain as previously described [18]. Amplicons were sequenced commercially (Macrogen, Seoul, Korea). The analyzed amino acid sequences were compared to the published tcdC sequence for strain VPI10463 [18].

\section{Antimicrobial susceptibility testing}

Antimicrobial susceptibility tests were performed with 120 C. difficile isolates using 10 randomly selected isolates per hospital and the agar dilution method on Brucella blood agar according to the recommendations of the CLSI [19]. Quality control strains used for susceptibility testing included Bacteroides thetaiotaomicron (ATCC 29741) and B. fragilis (ATCC 25285). Antimicrobial agents used were ampicillin (Sigma-Aldrich Co., St. Louis, MO, USA), piperacillin and tazobactam (Yuhan, Seoul, Korea), cefoxitin (Merck Sharp \& Dohme, West Point, PA, USA), cefotetan (Daiichi Pharmaceutical, Tokyo, Japan), clindamycin (Korea Upjohn, Seoul, Korea), imipenem and metronidazole (Choong Wae, Seoul, Korea), moxifloxacin (Bayer Korea, Seoul, Korea), and vancomycin (Chong Kun Dang, Seoul, Korea). For the combination of piperacillin and tazobactam, a constant amount of tazobactam (final concentration, $4 \mu \mathrm{g} / \mathrm{mL}$ ) was added to piperacillin. The CLSI breakpoints were used for the analysis. However, the CLSI guidelines do not recommend a breakpoint for VAN, and therefore the breakpoint suggested by the European Committee on Antimicrobial Susceptibility Testing (EUCAST; www.escmid.org/research_projects/ eucast) was used.

\section{RESULTS}

\section{Toxin analysis by PCR}

Of the total 408 isolates, 337 (82.6\%) were toxigenic $C$. difficile $\left(\mathrm{A}^{+} \mathrm{B}^{+}\right.$and $\left.\mathrm{A}^{-} \mathrm{B}^{+}\right)$. We identified $232(56.9 \%) \mathrm{A}^{+} \mathrm{B}^{+}$ strains and $105(25.7 \%) \mathrm{A}^{-} \mathrm{B}^{+}$strains. The recovery rates of the toxigenic strains were $70-100 \%$ according to the hospitals studied. The proportion of $\mathrm{A}^{-} \mathrm{B}^{+}$strains differed between the hospitals during the study period (from 0\% to $37.9 \%)$.

Twenty-nine (7.1\%) strains were $\mathrm{CDT}^{+}$. The proportion of $\mathrm{CDT}^{+}$strains varied between the hospitals (from $0 \%$ to

Table 1. The frequencies of the occurrences of toxins A-, B-, and binary toxin-producing strains in 12 South Korean hospitals

\begin{tabular}{|c|c|c|c|c|c|c|c|}
\hline \multirow{2}{*}{ Hospitals } & \multirow{2}{*}{$\mathrm{N}$ of isolates tested } & \multirow{2}{*}{ Study period } & \multirow{2}{*}{ Number of beds* } & \multicolumn{4}{|c|}{$N(\%)$} \\
\hline & & & & $\mathrm{A}^{+} \mathrm{B}^{+} \mathrm{CDT}^{-}$ & $\mathrm{A}^{+} \mathrm{B}^{+} \mathrm{CDT}^{+}$ & $\mathrm{A}^{-} \mathrm{B}^{+} \mathrm{CDT}^{-}$ & $\mathrm{A}^{-\mathrm{B}} \mathrm{CDT}$ \\
\hline Seoul A & 145 & Jan.2007-Dec. 2007 & 2,064 & $52(35.9)$ & $8(5.5)$ & 55 (37.9) & $30(20.7)$ \\
\hline Seoul B & 37 & Jan.2007-Jun.2008 & 758 & $19(51.4)$ & $4(10.8)$ & $9(24.3)$ & $5(13.5)$ \\
\hline Seoul C & 30 & Jan.2007-Feb.2008 & 938 & $13(43.3)$ & $6(20.0)$ & $6(20.0)$ & $5(16.7)$ \\
\hline Seoul D & 20 & Jan.2008-Feb.2008 & 2,200 & $8(40.0)$ & $3(15.0)$ & $3(15.0)$ & $6(30.0)$ \\
\hline Gyeonggi B & 17 & Jan. 2008-May. 2008 & 920 & $14(82.3)$ & $1(5.9)$ & $2(11.8)$ & $0(0)$ \\
\hline Gyeonggi C & 15 & Jan.2006-Dec. 2006 & 550 & $8(53.3)$ & $0(0)$ & $3(20.0)$ & $4(26.7)$ \\
\hline Chungnam & 22 & Oct.2007-May.2008 & 803 & $12(54.5)$ & $2(9.1)$ & $6(27.3)$ & $2(9.1)$ \\
\hline Daejeon & 25 & Mar.2008-Jun.2008 & 813 & $17(68.0)$ & $2(8.0)$ & $3(12.0)$ & $3(12.0)$ \\
\hline Busan & 20 & Feb.2007-Dec. 2007 & 912 & $12(60.0)$ & $0(0)$ & $6(30.0)$ & $2(10.0)$ \\
\hline Gwangju & 20 & Mar.2008-Jun.-2008 & 555 & $15(75.0)$ & $0(0)$ & $0(0)$ & $5(25.0)$ \\
\hline Gangwon & 16 & Nov.2007-May.2008 & 816 & $10(62.5)$ & $1(6.3)$ & $3(18.8)$ & $2(12.5)$ \\
\hline
\end{tabular}

*data from the Korean Hospital Association 2007.

Abbreviations: $A^{+} B^{+}$, toxin A-positive, toxin B-positive; $A^{-} B^{+}$, toxin A-negative, toxin B-positive; $A^{-} B$; toxin A-negative, toxin B-negative, $C D T^{+}$, binary toxin-positive; $\mathrm{CDT}^{-}$, binary toxin-negative. 


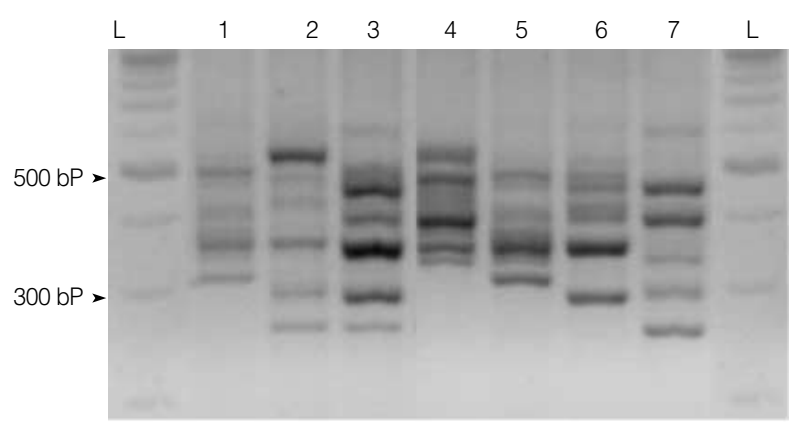

Fig. 1. PCR ribotype patterns of the Clostridium difficile isolates representing PCR ribotypes $A B 24, C 11, C 5, C 2, A B 14, A B 23$, and $\mathrm{aB}$ (Lane 1 to 7 , respectively). Lane $\mathrm{L}$ refers to $100 \mathrm{bp}$ ladder. Banding patterns of the $\mathrm{C} 5, \mathrm{C} 2$, and $\mathrm{aB}$ ribotypes were identical to the pattern of $C$. difficile ribotype 027,078 , and 017 strains.

20.0\%). All $\mathrm{CDT}^{+}$strains were $\mathrm{A}^{+} \mathrm{B}^{+}$(Table 1 ).

\section{PCR ribotyping}

A total of 50 different ribotype patterns were found. We identified 24 patterns of $\mathrm{A}^{+} \mathrm{B}^{+} \mathrm{CDT}^{-}$strains (ribotype $\mathrm{AB1} 1-\mathrm{AB} 24$ ), $12 \mathrm{~A}^{+} \mathrm{B}^{+} \mathrm{CDT}^{+}$(ribotype $\mathrm{C} 1-\mathrm{C} 12$ ), and $13 \mathrm{~A}^{-} \mathrm{B}^{-}$ (ribotype ab1-ab13). The PCR ribotypes aB, C5, and C2 are equivalent to the PCR ribotypes 017, 027, and 078 by O’Neill's method, respectively [17] (Fig. 1).

All $\mathrm{A}^{-} \mathrm{B}^{+}$strains showed the same banding pattern (ribotype $\mathrm{aB}$ ) in ribotyping, which was identical to the pattern of the $C$. difficile 1470 strain (ribotype 017). Ribotype aB was the predominant type (105 isolates, 25.7\%). The most frequently observed ribotypes of $C$. difficile in decreasing order were as follows: ribotype AB2 (71, 17.4\%), ribotype $\mathrm{AB} 3$ (30, 7.4\%), ribotype $\mathrm{AB} 1$ (27, 6.6\%), and ribotype AB17 (20, 4.9\%). These 5 ribotypes comprised $62.0 \%$ of the total. Ribotypes of $C$. difficile isolates were different from their toxin statuses.

Only 1 C. difficile isolate showed a pattern (ribotype C5) identical to PCR ribotype 027. Sequence analysis of tcdC in this isolate showed a single-base pair deletion at position 117 as well as a well-documented 18-bp deletion, which was identical to the sequence results of the epidemic strain of $C$. difficile 027. When examined by the E-test, the isolate was susceptible to moxifloxacin (MIC=0.5 $\mu \mathrm{g} / \mathrm{mL}$ ).

Thirteen strains of PCR ribotype 078 (ribotype C2) were identified in 6 hospitals, making ribotype 078 the most
Table 2. The MICs of 10 antimicrobial agents for 120 Korean Clostridium difficile isolates

\begin{tabular}{lccrrrr}
\hline \multirow{2}{*}{ Antimicrobials } & \multicolumn{3}{c}{$\mathrm{MIC}(\mu \mathrm{g} / \mathrm{mL})$} & & & $\mathrm{R}$ \\
\cline { 2 - 4 } & Range & $\mathrm{MIC}_{50}$ & $\mathrm{MIC}_{90}$ & & $\mathrm{I}$ & $\mathrm{R}$ \\
\hline Ampicillin & $1-8$ & 2 & 2 & 0 & 49 & 51 \\
Piperacillin & $2-64$ & 8 & 16 & 99 & 1 & 0 \\
Piperacillin- & $4-64$ & 8 & 16 & 99 & 1 & 0 \\
$\quad$ tazobactam & & & & & & \\
Cefoxitin & $64->128$ & 128 & $>128$ & 0 & 0 & 100 \\
Cefotetan & $8->128$ & 32 & 128 & 26 & 40 & 34 \\
Clindamycin & $0.5->128$ & 128 & $>128$ & 11 & 29 & 60 \\
Imipenem & $2-64$ & 8 & 16 & 22 & 53 & 25 \\
Metronidazole & $0.12-8$ & 1 & 4 & 100 & 0 & 0 \\
Moxifloxacin & $1->128$ & 2 & 16 & 53 & 5 & 42 \\
Vancomycin & $0.25-2$ & 0.5 & 1 & 100 & 0 & 0 \\
\hline
\end{tabular}

Abbreviations: MIC, minimum inhibitory concentration; S, Susceptible; I, Intermediate; R, Resistant.

prevalent ribotype among $\mathrm{CDT}^{+}$strains (13/29 $\mathrm{CDT}^{+}$strains, $44.8 \%$; $13 / 408$ isolates, $3.1 \%$ ).

\section{Antimicrobial susceptibility testing}

The in vitro activities of antimicrobial agents against C. difficile isolates are summarized in Table 2. No isolates were susceptible to cefoxitin and all except 1 were susceptible to piperacillin and piperacillin-tazobactam. The resistance rates to imipenem, cefotetan, moxifloxacin, ampicillin, and clindamycin were 25\%, 34\%, 42\%, 51\%, and $60 \%$, respectively. All strains were susceptible to metronidazole and vancomycin.

\section{DISCUSSION}

We conducted this study to enhance the knowledge on the nationwide epidemiology of $C$. difficile. This study included data from 12 hospitals in 7 different areas of South Korea.

The prevalence of $\mathrm{A}^{-} \mathrm{B}^{+}$strains differs according to the country studied. In Europe, $6.2 \%$ of toxigenic $C$. difficile isolates recovered in 2005 were $\mathrm{A}^{-} \mathrm{B}^{+}$[10]. In a recent study, $\mathrm{A}^{-} \mathrm{B}^{+}$strains comprised $33.3 \%$ of 75 toxigenic isolates from Shanghai and 0\% of 80 isolates from Stockholm [20]. The prevalence of $\mathrm{A}^{-} \mathrm{B}^{+}$strains was $25.7 \%(0-$ $37.9 \%$, according to the data obtained from hospitals) in 
this study. In our previous study, the prevalence of $\mathrm{A}^{-} \mathrm{B}^{+}$ strains increased steadily (4.2\% in 1995, 39.6\% in 2004) [21], and in another multicenter study conducted in Korea, 17.6-54.8\% of the isolated strains were $\mathrm{A}^{-} \mathrm{B}^{+}$in 2005 [22]. The prevalence of $\mathrm{A}^{-} \mathrm{B}^{+}$strains in Korea and Shanghai was much higher than in European countries.

The prevalence of $\mathrm{CDT}^{+}$strains was $7.1 \%(0-20.0 \%)$ in this study. Before the epidemics caused by ribotype 027, a binary toxin was identified in about $6 \%$ of clinical $C$. difficile isolates obtained in the United States and Europe $[16,23]$. The prevalence of $\mathrm{CDT}^{+} C$. difficile strains increased to $34.6 \%$ due to the ribotype 027 epidemics in Canada [24]. In our previous study, the prevalence of $\mathrm{CDT}^{+}$strains increased from 0\% in 2003 to 3.9\% in 2006 [21]. Therefore, we thought the prevalence of $\mathrm{CDT}^{+}$strains had steadily increased without evidence of a $C$. difficile epidemic. All $\mathrm{CDT}^{+}$strains were $\mathrm{A}^{+} \mathrm{B}^{+}$. Therefore, no additional binary toxin test was required for the diagnosis of CDAD.

A total of 408 C. difficile isolates were successfully typed with our PCR ribotyping method. Predominant ribotypes among the participating hospitals were not significantly different.

All $105 \mathrm{~A}^{-} \mathrm{B}^{+}$strains showed the same ribotyping pattern (aB), which was the most common ribotype (105/408, 25.7\%) and indistinguishable from the pattern of $C$. difficile 1470 (ribotype 017). It was previously reported that most $\mathrm{A}^{-} \mathrm{B}^{+}$ strains yield this distinct ribotype pattern in many studies, suggesting a worldwide clonal spread [7, 10, 21].

Only 1 PCR ribotype 027 strain was identified in hospital Seoul A. In contrast to epidemic 027 strains resistant to fluoroquinolone, this isolate was susceptible, which is in accordance with a report on 027 isolates obtained before 2001 in North America [11].

PCR ribotype 078 is the predominant ribotype in calves and pigs, and is an emerging new hypervirulent strain [25]. The prevalence of CDAD caused by a PCR ribotype 078 strain increased from 3\% to 13\% during 2005-2008 in The Netherlands. CDAD caused by type 078 strains has a similar severity of CDAD caused by type 027 strains [26]. Thirteen strains of PCR ribotype 078 were identi- fied in our study, which was the most prevalent ribotype among $\mathrm{CDT}^{+}$strains $\left(44.8 \%\right.$ of $\mathrm{CDT}^{+}$strains, $3.1 \%$ of all isolates).

Antimicrobial therapy plays a central role in the development of CDAD. The increasing use of fluoroquinolones in US health care facilities may have provided a selective advantage for the fluoroquinolone-resistant 027 strain and promoted its widespread emergence [11]. MTZ and VAN remain the most active agents in this study. No resistance to piperacillin-tazobactam was found in isolates from Shanghai and Stockholm [20] and only 1 non-toxigenic isolate showed intermediate resistance in this study. Resistance to other antimicrobials varies widely between countries [29]. The resistance rate to moxifloxacin was $42 \%$ in our study, which was lower than that in Scotland $(87.5 \%, 2007)$ and higher than that in Sweden $(15.0 \%$, 2009). The resistance rate to clindamycin was $60 \%$ in our study, which was lower than in Canada (90.9\%, 2009) and higher than that in Hungary $(27.5 \%, 2009)$ [27].

The MICs of ampicillin, piperacillin, piperacillintazobactam, cefoxitin, cefotetan, imipenem, metronidazole, and vancomycin were not significantly different according to the toxin status. However, the MIC50 values of clindamycin and moxifloxacin in $\mathrm{A}^{-} \mathrm{B}^{+}$strains were significantly higher than those of $\mathrm{A}^{+} \mathrm{B}^{+}$strains: 128 and 16 in $\mathrm{A}^{-} \mathrm{B}^{+}$versus 4 and 1 in $\mathrm{A}^{+} \mathrm{B}^{+}$, respectively (data not shown). It was reported that higher MICs of antimicrobial agents for predominant $C$. difficile strains may have played a role in their persistence and dissemination in hospitals [28, 29]. Therefore, the increased prevalence of $\mathrm{A}^{-} \mathrm{B}^{+}$strains in this study may reflect their higher MICs and the selective advantage it allows. This is the first nationwide study on the toxigenic status, including molecular genotyping and antimicrobial susceptibility pattern, of $C$. difficile isolates in South Korea. The prevalence of $\mathrm{A}^{-} \mathrm{B}^{+}$and $\mathrm{CDT}^{+}$strains was $25.7 \%$ and $7.1 \%$, respectively. Surveys of all $\mathrm{A}^{-} \mathrm{B}^{+}$strains showed that the most common ribotype was ribotype 017. We isolated 1 ribotype 027 strain, which is regarded as a historic isolate, with susceptibility to moxifloxacin. The prevalence of ribotype 078 was $3.1 \%$, which was higher than that of 
ribotype 027. We did not isolate strains with decreased susceptibility to MTZ or VAN, since these 2 antimicrobial agents can be used without an antimicrobial susceptibility test.

\section{ACKNOWLEDGEMENTS}

We thank Gwanghee Byun (Kyunghee University, Yongin, Korea) for laboratory assistance.

\section{REFERENCES}

1. Bartlett JG. Clostridium difficile: history of its role as an enteric pathogen and the current state of knowledge about the organism. Clin Infect Dis 1994;18(S4):S265-72.

2. Borriello SP, Davies HA, Kamiya S, Reed PJ, Seddon S. Virulence factors of Clostridium difficile. Rev Infect Dis 1990;12(S2):S185-91.

3. Braun V, Hundsberger T, Leukel P, Sauerborn M, von EichelStreiber C. Definition of the single integration site of the pathogenicity locus in Clostridium difficile. Gene 1996;181:29-38.

4. Hammond GA and Johnson JL. The toxigenic element of Clostridium difficile strain VPI 10463. Microb Pathog 1995;19:203-13.

5. Borriello SP, Wren BW, Hyde S, Seddon SV, Sibbons P, Krishna MM, et al. Molecular, immunological, and biological characterization of a toxin A-negative, toxin B-positive strain of Clostridium difficile. Infect Immun 1992;60:4192-9.

6. Lyerly DM, Barroso LA, Wilkins TD, Depitre C, Corthier G. Characterization of a toxin A-negative, toxin B-positive strain of Clostridium difficile. Infect Immun 1992;60:4633-9.

7. Alfa MJ, Kabani A, Lyerly D, Moncrief S, Neville LM, Al-Barrak A, et al. Characterization of a toxin A-negative, toxin B-positive strain of Clostridium difficile responsible for a nosocomial outbreak of Clostridium difficile-associated diarrhea. J Clin Microbiol 2000;38:2706-14.

8. Popoff MR, Rubin EJ, Gill DM, Boquet P. Actin-specific ADP-ribosyltransferase produced by a Clostridium difficile strain. Infect Immun 1988;56:2299-306.

9. Perelle S, Gibert M, Bourlioux P, Corthier G, Popoff MR. Production of a complete binary toxin (actin-specific ADP-ribosyltransferase) by Clostridium difficile CD196. Infect Immun 1997;65:1402-7.

10. Barbut F, Mastrantonio P, Delmée M, Brazier J, Kuijper E, Poxton I.. Prospective study of Clostridium difficile infections in Europe with phenotypic and genotypic characterisation of the isolates. Clin Microbiol Infect 2007;13:1048-57.

11. McDonald LC, Killgore GE, Thompson A, Owens RC Jr, Kazakova SV, Sambol SP, et al. An epidemic, toxin gene-variant strain of Clostridium difficile. N Engl J Med 2005;353:2433-41.

12. Warny M, Pepin J, Fang A, Killgore G, Thompson A, Brazier J, et al. Toxin production by an emerging strain of Clostridium difficile associated with outbreaks of severe disease in North America and Europe. Lancet 2005;366:1079-84.

13. Baines SD, O'Connor R, Freeman J, Fawley WN, Harmanus C, Mastrantonio $P$, et al. Emergence of reduced susceptibility to metronidazole in Clostridium difficile. J Antimicrob Chemother 2008;62: 1046-52.

14. Muto CA, Pokrywka M, Shutt K, Mendelsohn AB, Nouri K, Posey $\mathrm{K}$, et al. A large outbreak of Clostridium difficile-associated disease with an unexpected proportion of deaths and colectomies at a teaching hospital following increased fluoroquinolone use. Infect Control Hosp Epidemiol 2005;26:273-80.

15. Kato H, Kato N, Watanabe K, Iwai N, Nakamura H, Yamamoto T, et al. Identification of toxin A-negative, toxin B-positive Clostridium difficile by PCR. J Clin Microbiol 1998;36:2178-82.

16. Stubbs S, Rupnik M, Gibert M, Brazier J, Duerden B, Popoff M. Production of actin-specific ADP-ribosyltransferase (binary toxin) by strains of Clostridium difficile. FEMS Microbiol Lett 2000;186:307-12.

17. O’Neill GL, Ogunsola FT, Brazier JS, Duerden BI. Modification of a PCR ribotyping method for application as a routine typing scheme for Clostridium difficile. Anaerobe 1996;2:205-9.

18. Spigaglia P and Mastrantonio P. Comparative analysis of Clostridium difficile clinical isolates belonging to different genetic lineages and time periods. J Med Microbiol 2004;53:1129-36.

19. Clinical and Laboratory Standards Institute. Methods for antimicrobial susceptibility testing of Anaerobic bacteria; Approved standard. 7th ed. CLSI document M11-A7. Wayne, PA: Clinical and Laboratory Standards Institute, 2007.

20. Huang H, Fang H, Weintraub A, Nord CE. Distinct ribotypes and rates of antimicrobial drug resistance in Clostridium difficile from Shanghai and Stockholm. Clin Microbiol Infect 2009;15:1170-3.

21. Kim H, Riley TV, Kim M, Kim CK, Yong D, Lee K, et al. Increasing prevalence of toxin A-negative, toxin B-positive isolates of Clostridium difficile in Korea: impact on laboratory diagnosis. J Clin Microbiol. 2008;46:1116-7. 
22. Shin BM, Kuak EY, Yoo HM, Kim EC, Lee K, Kang JO, et al. Multicentre study of the prevalence of toxigenic Clostridium difficile in Korea: results of a retrospective study 2000-2005. J Med Microbiol 2008;57:697-701.

23. Geric B, Rupnik M, Gerding DN, Grabnar M, Johnson S. Distribution of Clostridium difficile variant toxinotypes and strains with binary toxin genes among clinical isolates in an American hospital. J Med Microbiol 2004;53:887-94.

24. Martin H, Willey B, Low DE, Staempfli HR, McGeer A, Boerlin P, et al. Characterization of Clostridium difficile strains isolated from patients in Ontario, Canada, from 2004 to 2006. J Clin Microbiol 2008;46:2999-3004.

25. Keel K, Brazier JS, Post KW, Weese S, Songer JG. Prevalence of PCR ribotypes among Clostridium difficile isolates from pigs, calves, and other species. J Clin Microbiol 2007;45:1963-4.
26. Goorhuis A, Bakker D, Corver J, Debast SB, Harmanus C, Notermans DW, et al. Emergence of Clostridium difficile infection due to a new hypervirulent strain, polymerase chain reaction ribotype 078 . Clin Infect Dis 2008;47:1162-70.

27. Huang H, Weintraub A, Fang H, Nord CE. Antimicrobial resistance in Clostridium difficile. Int J Antimicrob Agents 2009;34:516-22.

28. Drudy D, Quinn T, O’Mahony R, Kyne L, O'Gaora P, Fanning S. High-level resistance to moxifloxacin and gatifloxacin associated with a novel mutation in gyrB in toxin-A-negative, toxin-B-positive Clostridium difficile. J Antimicrob Chemother 2006;58:1264-7.

29. John R and Brazier JS. Antimicrobial susceptibility of polymerase chain reaction ribotypes of Clostridium difficile commonly isolated from symptomatic hospital patients in the UK. J Hosp Infect 2005; 61:11-4. 